\title{
Searches for Supersymmetry with Photons at CMS
}

\author{
Si Xie* on behalf of the CMS Collaboration \\ California Institute of Technology \\ E-mail: sixie@hep.caltech.edu
}

\begin{abstract}
We present results on searches for supersymmetry with photons in the final state using pp collision at a center of mass energy of $13 \mathrm{TeV}$ collisions with the CMS experiment. Results for searches for excesses at high missing transverse energy in the diphoton and single photon final states are shown, as well as a search for anomalous Higgs boson production in the diphoton decay channel. We interpret the results in terms of limits on the production cross section for simplified models of gluino-pair and sbottom-pair production as well as chargino-neutralino production in the gauge mediation supersymmetry breaking scenario.
\end{abstract}

38th International Conference on High Energy Physics 3-10 August 2016

Chicago, USA

${ }^{*}$ Speaker. 
The recent increase to the center of mass energy of the Large Hadron Collider (LHC) from $8 \mathrm{TeV}$ to $13 \mathrm{TeV}$ motivates new searches for supersymmetry as the rate of production of heavy squarks and gluinos increased by an order of magnitude. Final states with energetic photons and large missing transverse energy emerge naturally in models of supersymmetry (SUSY) that is broken by gauge mediation. Typically the stable lightest supersymmetric particle (LSP) is the gravitino and is nearly massless. Moreover, the presence of photons in the final state suppresses the top quark pair production and $\mathrm{W}$ and $\mathrm{Z}$ boson production backgrounds that typically dominate alternative searches for SUSY involving multiple jets and missing transverse energy, and therefore serves to probe complementary phase space. Finally, the use of the Higgs boson decay to two photons is a powerful complementary signature in the search for SUSY.

We present several complementary searches for supersymmetry involving photon final states from the CMS experiment [1]. We interpret the results of the searches in terms of simplified models with fixed production and decay modes, and the models considered in searches involving photons are shown in Figure 1

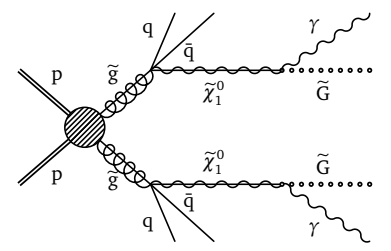

(a) T5gg

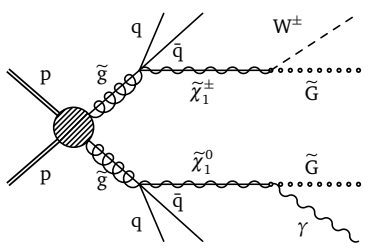

(b) $\mathrm{T} 5 \mathrm{Wg}$

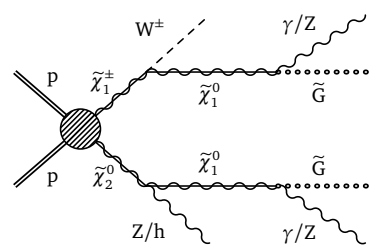

(c) $\tilde{\chi^{ \pm}} \tilde{\chi^{0}}$ production

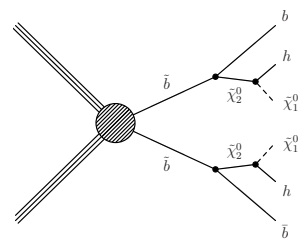

(d) $\tilde{b}$-pair production

Figure 1: The Feynman diagrams for several simplified models are presented. Gluino pair production decaying to two photons and a photon and a W boson are shown in (a) and (b). Chargino-Neutralino production is shown in (c), and sbottom pair production decaying to a final state with two b quarks and two Higgs bosons is shown in (d).

\section{Search using Two Photons and Missing Transverse Energy}

Using $2.3 \mathrm{fb}^{-1}$ of integrated luminosity from the 2015 dataset, this search[4] selects events with two central photons $(|\eta|<1.44)$ whose $p_{T}$ is larger than $40 \mathrm{GeV}$. Electrons are suppressed with a veto on pixel track seeds, and the missing transverse energy (MET) is required to be larger than $100 \mathrm{GeV}$. The search is performed in four exclusive MET bins: $(100,110)$; $(110,120)$; $(120,140)$; and $(140, \infty)$.

The dominant background is QCD diphoton production, where the MET is from instrumental sources, primarily jet mis-measurement. A data-driven method is used to estimate this background by using the The $Z \rightarrow$ ee control region to predict the shape of the MET distribution for the search region after using the Monte Carlo simulation to correct for the difference in the recoil spectrum, while a comparison of the shape to the control region with inverted photon identification or isolation requirements is used to estimate systematic uncertainties. The data and background prediction for the search region is shown in Figure 2, and no significant deviation is observed. Limits on the production cross section for gluino pairs decaying to two quarks, a photon, and the LSP is derived. Gluinos with masses below $1.65 \mathrm{TeV}$ are excluded, assuming $100 \%$ branching ratio to the decay mode considered. 

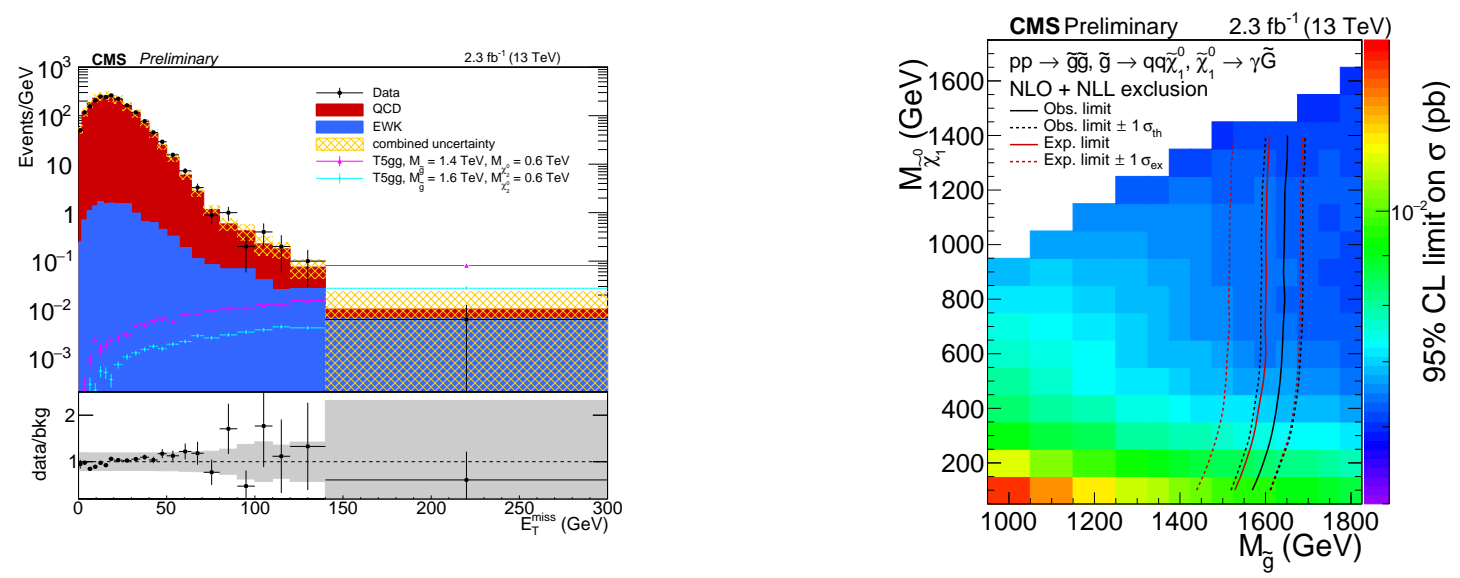

Figure 2: Left: The MET distribution in the signal region data is compared to the background prediction. The shaded regions show the systematic uncertainties on the background prediction and the ratio of data to predicted background. Right: The 95\% confidence level upper limits on the gluino pair production cross section as a function of gluino and neutralino masses. The contours show the observed and median expected exclusions assuming the NLO + NLL cross sections, with their one standard deviation uncertainties.

\section{Search using One Photon and Missing Transverse Energy}

A complementary search requiring one photon and MET is performed on the same dataset collected in 2015 [5]. The photon is required to have $p_{T}>180 \mathrm{GeV}$. The MET significance and the transverse mass $\left(M_{T}\right)$ formed by the photon and the MET are used to define the search and control regions as shown on the left of Figure 3. The search is performed in bins of the variable $S_{T}^{\gamma}$ defined as the scalar sum of the MET and the transverse momentum of the photon, above $600 \mathrm{GeV}$.
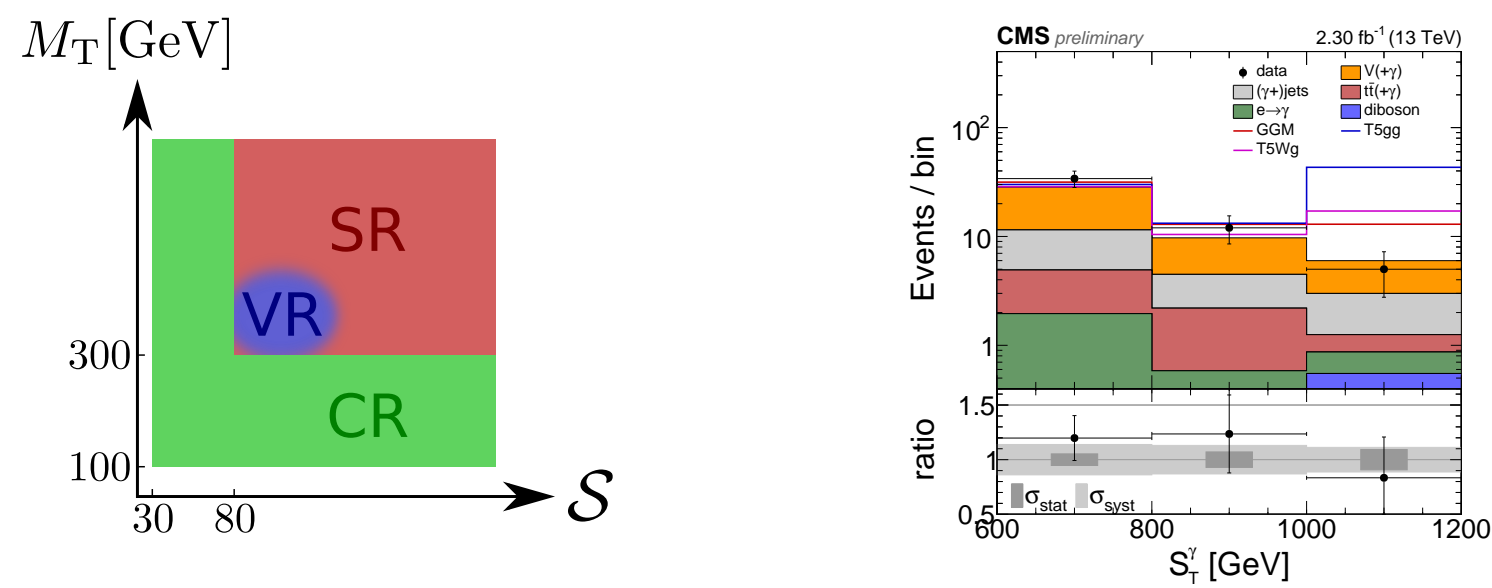

Figure 3: Left: Definitions of the search region (SR), control region (CR) and cross-check or verification region (VR). Right: Comparison of the search region data and the background prediction is shown along with the signal prediction for three hypothetical signal models.

The main backgrounds are $Z+\gamma$ where the $Z$ boson decays to a pair of neutrinos, $W+\gamma$ where the $\mathrm{W}$ boson decays to a lepton and neutrino, and $\gamma+$ jets. A template fit to the distribution of $\Delta \phi$ between the MET and the leading jet is performed in the control region in order to determine the 
scale factors that are required to correct the Monte Carlo simulation prediction. Correction factors of $1.46 \pm 0.13$ and $0.69 \pm 0.17$ were measured for the $\gamma+$ jets and $W / Z+\gamma$ processes. The background predictions were systematically validated in the control region with low MET significance and low $M_{T}$, the lepton $+\gamma$ control region, and the control region with $S_{T}^{\gamma}<600 \mathrm{GeV}$, and exhibit excellent agreement as shown in Figure 4

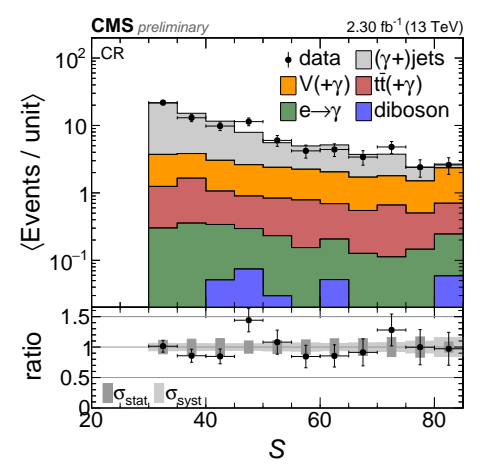

(a) Low MET Significance and Low $M_{T}$ Control Region

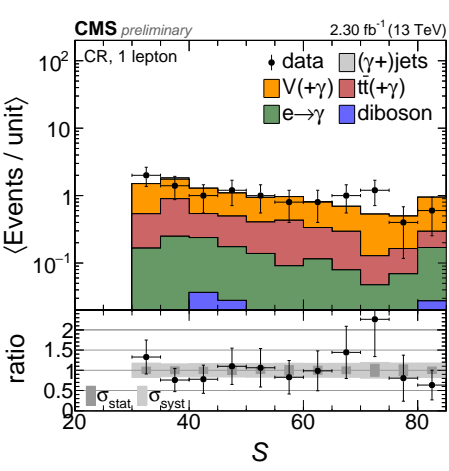

(a) Lepton $+\gamma$ Control Region

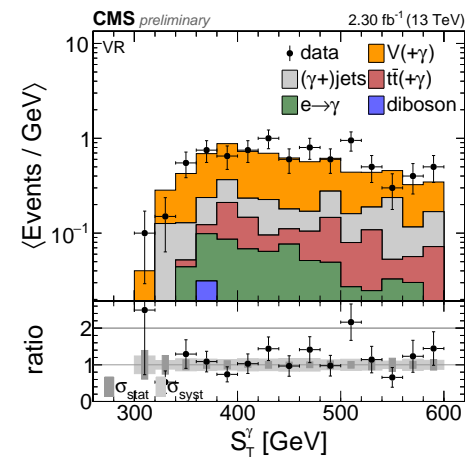

(a) Low $S_{T}^{\gamma}$ Control Region

Figure 4: The MET significance (S) and $S_{T}^{\gamma}$ distributions in data is compared to the background prediction in several background dominated control regions.

The search region data is compared to the background prediction on the right of Figure 3 , and no significant deviation is observed. We interpret the result in terms of upper limits on the cross section for chargino-neutralino production as a function of the Wino $(\tilde{W})$ and Bino $(\tilde{B})$ mass on the left of Figure 5, and on the cross section for gluino pair production decaying to a $\gamma \gamma$ or a $\mathrm{W}+\gamma$ final state in the center and right of Figure 5 respectively. We observe complementary sensitivity to the diphoton+MET search.
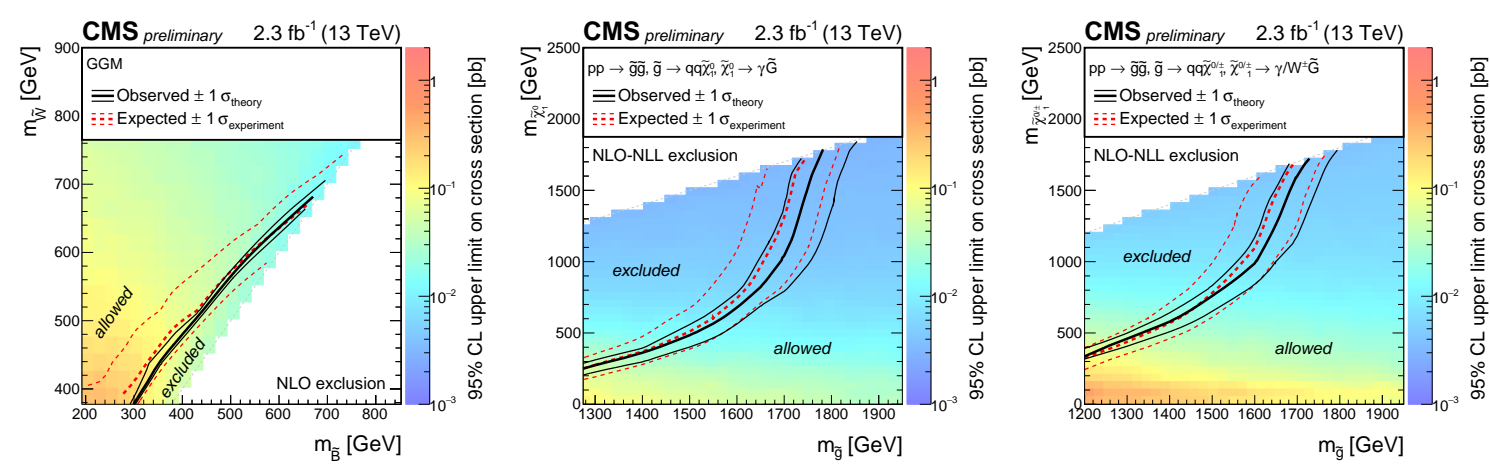

Figure 5: Left: The 95\% CL limits for chargino-neutralino production in the General Gauge Mediation model as a function of the bino and wino mass. Center: The 95\% CL limits for gluino pair production decaying to two quarks, a photon, and the LSP. Right: The 95\% CL limits for gluino pair production decaying to two quarks, a photon or a $\mathrm{W}$ boson with $50 \%$ branching ratio, and the LSP. The color scales encode the observed upper cross section limit for each point. The lines represent the observed (black) and expected (red) exclusion contours and their uncertainties. 


\section{Search using Higgs $\rightarrow \gamma \gamma$ and Razor Variables}

We search for an excess of Higgs production using the $\gamma \gamma$ decay channel in events with at least one additional jet using $15.2 \mathrm{fb}^{-1}$ of data collected in 2015 and 2016 [6]. Events are categorized according to the $p_{T}$ of the Higgs boson candidate, the presence of additional Higgs to $b \bar{b}$ or $\mathrm{Z}$ to $b \bar{b}$ candidates, and the estimated resolution of the diphoton pair. Events with Higgs $p_{T}$ above $110 \mathrm{GeV}$ is categorized as High-Pt; events with an additional Higgs to $b \bar{b}$ or $\mathrm{Z}$ to $b \bar{b}$ candidates is categorized as $\mathbf{H} / \mathbf{Z} \mathbf{b b}$; events with an estimated diphoton mass resolution below $0.85 \%$ is categorized as High-Res; and the remaining events are categorized as Low-Res. Within each category, the razor variables $M_{R}$ and $R^{2}[7,8]$ are used to define bins that improve signal to background discrimination. SUSY signal events tend to populate regions at larger values of $M_{R}$ and $R^{2}$. The bins used are summarized in Table 1.

Table 1: A summary of the search region bins in each category is presented.

\begin{tabular}{|c|c|c|c||c|c|c|c|}
\hline Bin Number & Category & $M_{R}(\mathrm{GeV})$ Bin & $R^{2}$ Bin & Bin Number & Category & $M_{R}(\mathrm{GeV}) \mathrm{Bin}$ & $R^{2} \mathrm{Bin}$ \\
\hline 0 & HighPt & $600-\infty$ & $0.025-\infty$ & 7 & HighPt & $600-1250$ & $0.015-0.025$ \\
1 & HighPt & $150-600$ & $0.130-\infty$ & 8 & H $(\gamma \gamma)$-H $/ \mathrm{Z}(\mathrm{bb})$ & $150-\infty$ & $0.000-\infty$ \\
2 & HighPt & $1250-\infty$ & $0.000-0.025$ & 9 & HighRes & $150-250$ & $0.000-0.175$ \\
3 & HighPt & $150-450$ & $0.000-0.130$ & 10 & HighRes & $150-250$ & $0.175-\infty$ \\
4 & HighPt & $450-600$ & $0.000-0.035$ & 11 & HighRes & $250-\infty$ & $0.05-\infty$ \\
5 & HighPt & $450-600$ & $0.035-0.130$ & 12 & HighRes & $250-600$ & $0.000-0.05$ \\
6 & HighPt & $600-1250$ & $0.000-0.015$ & 13 & HighRes & $600-\infty$ & $0.000-0.05$ \\
\hline
\end{tabular}

Within each bin, the signal and background are estimated by performing an unbinned fit to the diphoton mass distribution. The non-resonant backgrounds primarily from QCD diphoton production exhibit an exponentially decaying shape, while the Standard Model Higgs background and exotic signal models exhibit a peaking shape consistent with the Higgs resonance. The background shapes are modeled as either exponential functions or Bernstein polynomials, and are determined bin-by-bin using a combination of statistical tests for over-modeling and bias tests for appropriate coverage. The resonant shape is extracted from the Monte Carlo simulation separately for each bin. Example fits to the data are shown for a couple of bins in Figure 6. The background yield from the Standard Model Higgs production in each bin is constrained to the prediction from the Monte Carlo simulation. Systematic uncertainties due to missing higher order corrections, PDF uncertainties, photon selection efficiencies, jet energy scale, and b-tagging efficiency are all propagated and amount to $20-30 \%$ depending on the category and bin.

A summary of the observed statistical significance for all bins considered is shown on the left of Figure 7. We observe that the most significant excess occurs in "bin 0", in the High-Pt category with $M_{R}>600 \mathrm{GeV}$ and $R^{2}>0.025$, and has a local significance of $2.5 \sigma$. The search result is interpreted in terms of limits on the cross-section for sbottom-pair production with each sbottom decaying to a b quark, a Higgs boson, and the LSP, and are shown on the right of Figure 7 as a function of the sbottom mass and the LSP mass.

\section{References}

[1] CMS Collaboration, JINST 3 S08004 (2008). 

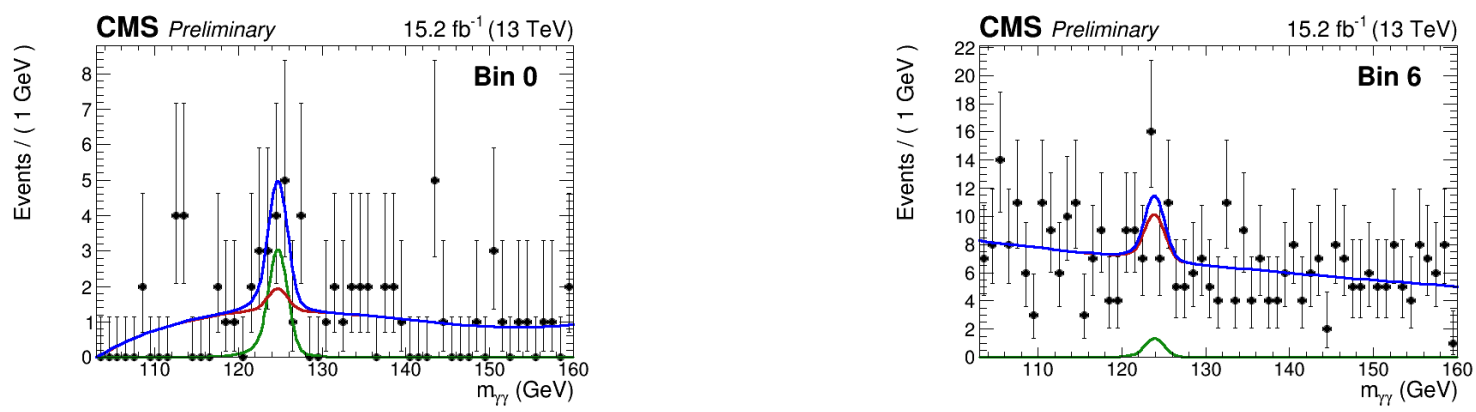

Figure 6: Search region data is fitted to a model that includes the non-resonant background, the Standard Model Higgs background, and an exotic Higgs signal. The blue curve represents the sum of all processes; the red curve is the sum of the background models; and the green curve represents the best fit exotic Higgs signal. Left: Bin0 is in the High-Pt category with $M_{R}>600 \mathrm{GeV}$ and $R^{2}>0.025$. Right: Bin6 is in the High-Pt category with $600 \mathrm{GeV}<M_{R}<1250 \mathrm{GeV}$ and $R^{2}<0.015$.
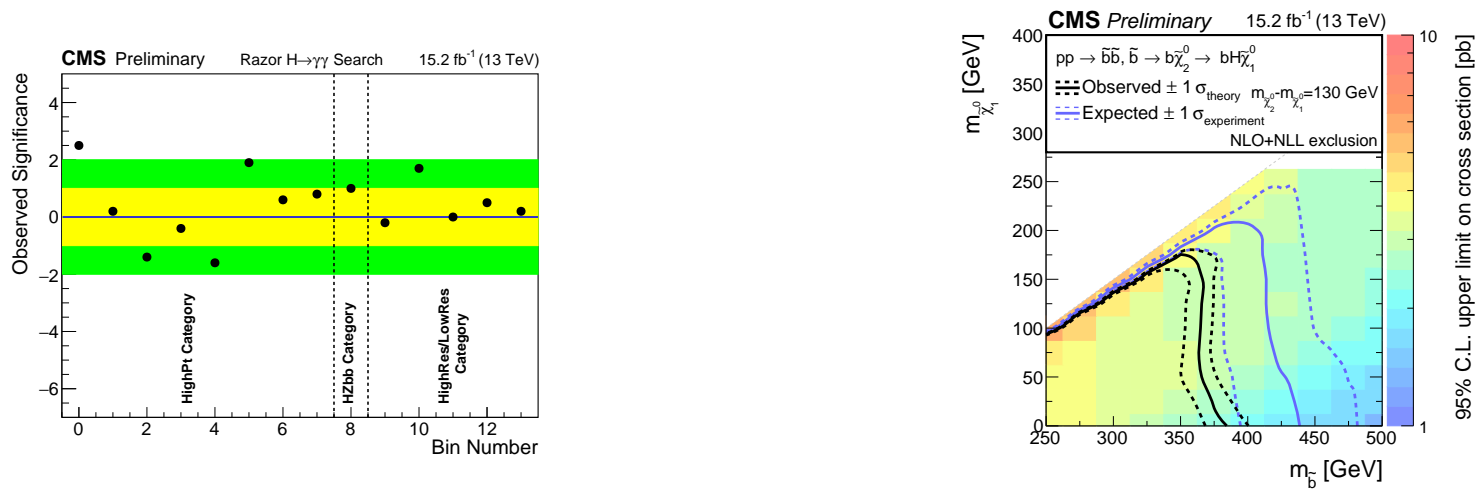

Figure 7: Left: Observed Local Significance is shown for each bin and category considered in the analysis. Right: The 95\% CL upper limits on the cross section for sbottom-pair production is shown as a function of the sbottom and LSP masses.

[2] P. Meade, N. Seiberg, and D. Shih, “General Gauge Mediation”, Prog. Theor. Phys. Suppl. 177 (2009) 143-158.

[3] M. Buican, P. Meade, N. Seiberg, and D. Shih, "Exploring General Gauge Mediation”, JHEP 0903 (2009) 016.

[4] CMS Collaboration, "Search for supersymmetry in events with photons and missing transverse energy", CMS-PAS-SUS-15-012.

[5] CMS Collaboration, "Search for supersymmetry in final states with at least one photon and $E_{T}^{\text {miss }}$ in pp collisions at $\sqrt{s}=13$ TeV”, CMS-PAS-SUS-16-023.

[6] CMS Collaboration, "Search for supersymmetry in events with a Higgs decaying to two photons using the razor variables", CMS-PAS-SUS-15-012.

[7] CMS Collaboration, "Inclusive search for squarks and gluinos in pp collisions at $\sqrt{s}=7$ TeV", Phys. Rev. D 85012004 (2012).

[8] CMS Collaboration, "Search for supersymmetry with razor variables in pp collisions at $\sqrt{s}=8 \mathrm{TeV}$ ", Phys. Rev. D 90112001 (2014). 